\title{
Robust MPC with recursive model update ${ }^{\star}$
}

\author{
Matthias Lorenzen $^{\text {a }}$, Mark Cannon ${ }^{\mathrm{b}}$, Frank Allgöwer ${ }^{\mathrm{a}}$ \\ anstitute for Systems Theory and Automatic Control, University of Stuttgart, Germany \\ ${ }^{\mathrm{b}}$ Department of Engineering Science, University of Oxford, United Kingdom
}

\begin{abstract}
Robust constrained control of linear systems with parametric uncertainty and additive disturbance is addressed. The main contribution is the introduction of a mathematically rigorous and computationally tractable framework for stabilizing model predictive control with online parameter estimation to improve performance and reduce conservatism. Requirements for closedloop stability and provable constraint satisfaction are considered separately, resulting in the use of online set-membership system identification combined with homothetic prediction tubes for robust constraint satisfaction, and an $\mathcal{H}_{\infty}$ optimal point estimate of the unknown parameters to achieve a finite closed-loop gain from the disturbance to the state. Extensions to timevarying parameters and persistently exciting inputs to guarantee parameter convergence are presented. A numerical example illustrates the proven properties and efficacy of the approach.
\end{abstract}

Key words: model predictive control, adaptive model predictive control, receding horizon control, control of constrained systems, adaptive control, uncertain linear systems, system identification

\section{Introduction}

Model predictive control (MPC) has become one of the main tools to handle multivariable constrained control problems. Given a model of the process, the basic idea is to solve an open-loop finite-horizon optimal control problem at each sampling time with the current state as initial condition. Since the performance and stability of MPC crucially depend on the model accuracy, robust and stochastic MPC has received much attention $[21,33]$. While these approaches are suitable for handling unmodeled dynamics and rapidly changing disturbances, they are inherently conservative for slowly changing or constant parametric uncertainty. To reduce the cost of manual tuning in MPC and cope with slowly changing dynamics, e.g. due to changing environment or wear and tear, there is a strong interest in self-tuning predictive control formulations but only few solution strategies are available $[25,28]$.

\footnotetext{
* M. Lorenzen and F. Allgöwer would like to thank the German Research Foundation (DFG) for financial support of the project within the Cluster of Excellence in Simulation Technology (EXC 310/2) at the University of Stuttgart.

Email addresses:

matthias.lorenzen@ist.uni-stuttgart.de (Matthias Lorenzen), mark.cannon@eng.ox.ac.uk (Mark Cannon), frank.allgower@ist. uni-stuttgart.de (Frank Allgöwer).
}

As MPC includes an explicit model of the process to be controlled, it should be amenable for online parameter adaption and system identification. The difficulty lies in finding a rigorous yet tractable formulation with provable constraint satisfaction and stability properties. Most system identification methods lack explicit error bounds or convergence guarantees when used for closedloop systems and in the presence of additive disturbance or measurement noise. Similarly, given a disturbance and uncertainty model, most robust MPC formulations rely on extensive offline computations and hence are not suitable for a changing uncertainty description.

As a result, the existing literature on the topic can be grouped into two main categories, depending on their respective focus. The first category subsumes research that focuses on system identification and online learning applied in MPC. In [24], the authors derive a persistence of excitation (PE) condition, which is introduced as an additional constraint in the MPC optimization, thereby ensuring parameter convergence in a recursive estimation scheme. In $[17,18]$, a Kalman Filter is employed for parameter estimation and sufficient excitation is achieved by explicitly incorporating the predicted covariance matrix in the cost. The result is a dual control scheme, which optimizes identification and regulation objectives simultaneously. Further advanced identification methods for nonlinear systems have been successfully combined with 
linear robust MPC and nonlinear MPC, e.g., Gaussian process regression $[20,27]$ or particle filtering [4].

The second category subsumes research that focuses on the MPC algorithm, in particular convergence of the state, constraint satisfaction and (recursive) feasibility of the online optimization. In $[2,11]$, a generic online system identification is used within a robust MPC framework. In both contributions, recursive feasibility and constraint satisfaction is guaranteed by employing robust MPC methods based on an a priori given uncertainty set that is not updated online. System identification is used only to update a nominal prediction model in order to increase the closed-loop performance.

A small number of publications combine the advantages of both approaches, namely online model adaption and provable closed-loop properties. In [19], stabilizing adaptive MPC for linear SISO systems based on a modified least squares estimation is introduced but the approach crucially relies on the assumption of noise and additive disturbances being absent. Results for nonlinear systems have been presented in $[1,15]$ using set-membership system identification, yet the algorithm leads to a computationally demanding, non-convex optimization program to be solved online. A computationally tractable combination of set-membership system identification and robust constraint tightening has been proposed in [34] and successfully applied to a building control example in [35]. However, the algorithm relies on uncertain FIR models and is hence restricted to stable systems. Furthermore, the approach thereby requires a long prediction horizon and the parametric uncertainty is described by a polytope in an high dimensional space, which increases the computational complexity. Recently, the algorithm has been extended in [9] with an additional linear Recursive Least Squares filter to handle chance constraints.

Building upon the results in [34] and [36], this paper introduces an adaptive MPC algorithm for linear systems with model uncertainty and additive disturbances, however, in a more general state-space formulation. In particular, a computationally tractable framework for MPC with online parameter adaption and guaranteed constraint satisfaction is presented. Requirements for robust constraint satisfaction and for closed-loop stability are considered separately. The former requires bounds on possible parameter and future state values, hence making set-membership estimation, as proposed in [34], combined with a set-membership prediction tube a suitable choice. For the latter, gains from the disturbance to the prediction error are most relevant, which leads to the additional use of a suitable point estimate. The state-space formulation makes it easy to incorporate extensions that have been developed for the non-adaptive case. The emphasis is put on setpoint stabilization, but the proposed combination of system identification and constraint tightening constitutes a solid basis for work on Dual MPC or tracking problems for systems with uncertain or slowly varying parameters.

The main limitation of the proposed approach is due to using an MPC formulation with terminal constraint and terminal cost. If the uncertain system is unstable, this requires the existence of a robustly stabilizing feedback law and a common Lyapunov function for the stabilized system, for all model parameters in a given prior bounding set. Compared to the classical literature on adaptive control, this assumption is restrictive. However, as a requirement of robust $\mathrm{MPC}$, it is in general unavoidable if state constraints are to be satisfied robustly. Similar assumptions are made in [1], while [34] is restricted to stable systems. A further limitation comes from the use of polytopes, which generally cause algorithm complexity to scale badly with the system dimension. However, the presented approach directly extends to using ellipsoids instead of polytopes; this approach would suffer increased conservativeness but its complexity would scale better.

More generally, the approach builds upon set-membership system identification, where pioneering results were published in the 60s for integral type bounds and, starting in the $80 \mathrm{~s}$, for componentwise bounds [3], as well as Tube MPC $[22,29]$, which is still an active research topic.

Preliminary results have been presented in the conference paper [23]. These results are extended and refined here by providing a set-membership update with a priori bounded complexity and we also discuss timevarying parameters and persistence of excitation. Most importantly, the algorithm has been extended to include a point-estimate and the cost function changed to a certainty-equivalence cost instead of a min-max cost. This allows to prove a finite $\ell_{2}$ gain, which is novel in the adaptive MPC literature.

The remainder is organized as follows. In Section 2 the main assumptions and problem setup are introduced. In Section 3 the system identification and constraint reformulation are presented, followed by a summary of the stabilizing adaptive MPC algorithm and its properties. Section 4 presents extensions to time-varying parameters and persistently exciting regressors to guarantee parameter convergence. A numerical example is presented in Section 5 and Section 6 provides some conclusions and directions for future work.

Notation The notation employed is standard. Uppercase letters are used for matrices and lower case for vectors. $[A]_{j}$ and $[a]_{j}$ denote the $j$-th row and entry of the matrix $A$ and vector $a$, respectively. By 1 we denote a column vector of ones and by $I$ the identity matrix of appropriate size, respectively. Positive (semi)definite matrices $A$ are denoted $A \succ 0(A \succeq 0)$. The Euclidean 
norm of a vector $x$ is denoted $\|x\|$ and for $A \succ 0$ we define $\|x\|_{A}^{2}=x^{\top} A x . \mathbb{R}_{\geq 0}$ is the set of the non-negative reals and $\mathbb{N}_{a}^{b}=\{n \in \mathbb{N} \mid a \leq n \leq b\}$. We use $x_{k}$ for the (real, measured) state at time $k$ and $x_{l \mid k}$ for the state predicted $l$ steps ahead at time $k . A \oplus B$ denotes the Minkowski set addition and $A \ominus B$ denotes the Pontryagin set difference. $\Pi_{A}(x)=\arg \min _{y \in A}\|x-y\|$ denotes the Euclidean projection of a vector $x \in \mathbb{R}^{n}$ onto a closed, nonempty set $A \subseteq \mathbb{R}^{n}$. $\mathbb{B}_{\varepsilon}(x)$ denotes an open ball of radius $\varepsilon$ centered on $x$.

\section{Problem formulation and preliminaries}

This paper considers the control of a discrete-time, linear system with state $x_{k} \in \mathbb{R}^{n}$, input $u_{k} \in \mathbb{R}^{m}$, additive disturbance $w_{k} \in \mathbb{W} \subseteq \mathbb{R}^{n}$, and unknown but constant ${ }^{1}$ parameter $\theta=\theta^{*} \in \overline{\mathbb{R}}^{p}$

$$
x_{k+1}=A(\theta) x_{k}+B(\theta) u_{k}+w_{k} .
$$

We make the following assumption on the disturbance and uncertainty.

\section{Assumption 1 (Disturbance and Uncertainty)}

The disturbance set $\mathbb{W}$ is a bounded, convex polytope given by

$$
\mathbb{W}=\left\{w \in \mathbb{R}^{n} \mid H_{w} w \leq \mathbf{1}\right\}
$$

with $H_{w} \in \mathbb{R}^{q_{w} \times n}$ and $\mathbf{1} \in \mathbb{R}^{q_{w}}$. The system matrices depend affinely on the parameter vector $\theta \in \mathbb{R}^{p}$

$$
(A(\theta), B(\theta))=\left(A_{0}, B_{0}\right)+\sum_{i=1}^{p}\left(A_{i}, B_{i}\right)[\theta]_{i}
$$

and a bounded parameter set given by

$$
\Theta=\left\{\theta \in \mathbb{R}^{p} \mid H_{\theta} \theta \leq h_{\theta}\right\},
$$

which contains the true parameter vector $\theta^{*}$ is known.

To capture actuator saturation or restrict the state to desirable operating regions, the controller must enforce mixed state and input constraints that can be modeled with linear inequalities. The state and inputs are assumed to be constrained to a bounded polytopic set $\mathbb{Z}$ given by

$$
\mathbb{Z}=\left\{(x, u) \in \mathbb{R}^{n} \times \mathbb{R}^{m} \mid F x+G u \leq \mathbf{1}\right\}
$$

with given matrices $F \in \mathbb{R}^{c \times n}$ and $G \in \mathbb{R}^{c \times m}$.

The control objective is to find a stabilizing control law for system (1) such that the constraints $(x, u) \in \mathbb{Z}$ are satisfied robustly and the model uncertainty $(3)$ is taken into account and updated consistently with the state and input history.

\footnotetext{
1 An extension to time-varying parameters in presented in Section 4.1 .
}

Indirect adaptive MPC To solve the control problem, a Tube MPC algorithm with online parameter estimation is considered, in which the requirements for stability and robust constraint satisfaction are treated separately. Specifically, for the former, a nominal estimate $\hat{\theta}_{k}$ is employed in a certainty equivalence approach. For the latter, a bounding set $\Theta_{k}$ for the unknown parameter $\theta$ is updated consistently with the prior set $\Theta$ and the state and input trajectories $\left\{x_{i}, u_{i}\right\}_{i \in \mathbb{N}_{0}^{k}}$ observed up to time $k$.

The bounding set $\Theta_{k}$ is employed to determine an admissible state tube $\left\{\mathbb{X}_{l \mid k}\right\}_{l \in \mathbb{N}_{0}^{N}}$, i.e., a sequence of sets $\mathbb{X}_{l \mid k} \subset \mathbb{R}^{n}$ with $l=0, \ldots, N$ that provides an outer bound for the predicted states and satisfies the constraints (4). Specifically, let

$$
\begin{aligned}
& \mathbb{X}_{0 \mid k} \ni x_{k}, \\
& \mathbb{X}_{l+1 \mid k} \ni A(\theta) x+B(\theta) u_{l \mid k}(x)+w \\
& \quad \forall x \in \mathbb{X}_{l \mid k}, w \in \mathbb{W}, \theta \in \Theta_{k}, \\
& x \times u_{l \mid k}(x) \in \mathbb{Z} \quad \forall x \in \mathbb{X}_{l \mid k}
\end{aligned}
$$

with some input parametrization $u_{l \mid k}: \mathbb{R}^{n} \rightarrow \mathbb{R}^{m}$.

A feedback law is then determined by a receding horizon scheme. At each sampling time $k$, a finite horizon optimal control problem is solved, given by

$$
\begin{array}{r}
V_{N}\left(x_{k}, \hat{\theta}_{k}, \Theta_{k}\right)=\min _{\mathbf{u}_{N \mid k}} J\left(x_{k}, \hat{\theta}_{k}, \mathbf{u}_{N \mid k}\right) \\
\text { s.t. }(5), \mathbb{X}_{N \mid k} \subseteq \mathbb{X}_{f}
\end{array}
$$

with a cost function $J$ to be defined, horizon length $N$, and terminal set $\mathbb{X}_{f}$. Assuming a minimizer $\mathbf{u}_{N \mid k}^{*}=$ $\left\{u_{l \mid k}^{*}\right\}_{l \in \mathbb{N}_{0}^{N-1}}$ exists, the control law is defined by $u\left(x_{k}\right)=$ $u_{0 \mid k}^{*}$.

In the following, we introduce a suitable choice for the system identification and tube parametrization to obtain a computationally tractable optimization problem. This leads to a basic framework to guarantee recursive feasibility and closed-loop constraint satisfaction in a receding horizon control algorithm. Furthermore, we show that a standard quadratic cost with a suitably chosen gradient-based algorithm for parameter point estimation leads to a finite gain property from the additive disturbance to the state $x_{k}$.

\section{Robust adaptive MPC}

In this section, first a suitable parameter estimation scheme is introduced, followed by a computationally tractable parametrization for an admissible state tube $\left\{\mathbb{X}_{l \mid k}\right\}_{l \in \mathbb{N}_{0}^{N}}$. Thereafter, a cost function and terminal constraint for a stabilizing MPC scheme is presented. 
Finally, the section concludes with a summary of the MPC algorithm and an analysis of system theoretic properties of the closed loop.

\subsection{Parameter estimation}

Many parameter estimation schemes have been presented in the literature but our main concern here is to define a parameter estimation scheme that provides the desirable system theoretic properties and still enables a computationally tractable MPC algorithm. In order to non-conservatively guarantee recursive feasibility and constraint satisfaction, the parameter estimation is based upon a set-membership estimation, which at each time step yields a polytopic parameter bounding set. Furthermore, to ensure a finite closed-loop gain from the additive disturbance to the system state $x_{k}$, an additional point estimate for the unknown parameter $\theta^{*}$ is employed. We introduce a set-membership system identification for the state space description (1), (2) and a projected least mean squares algorithm to update point estimates of the parameter vector.

Bounding set Let $D(x, u) \in \mathbb{R}^{n \times p}$ be defined by

$D(x, u)=\left[A_{1} x+B_{1} u, A_{2} x+B_{2} u, \ldots, A_{p} x+B_{p} u\right]$

$D_{k}=D\left(x_{k}, u_{k}\right)$, and $d_{k}=A_{0} x_{k-1}+B_{0} u_{k-1}-x_{k}$. At time $k \geq 1$, given $x_{k-1}, u_{k-1}$, and $x_{k}$, the non-falsified parameter set can be described by

$$
\begin{aligned}
\Delta_{k} & =\left\{\theta \in \mathbb{R}^{p} \mid x_{k}-\left(A(\theta) x_{k-1}+B(\theta) u_{k-1}\right) \in \mathbb{W}\right\} \\
& =\left\{\theta \in \mathbb{R}^{p} \mid-H_{w} D_{k-1} \theta \leq \mathbf{1}+H_{w} d_{k}\right\} .
\end{aligned}
$$

With this, the membership set of the uncertain parameter $\theta$ at time $k$ is given by

$$
\Theta_{k}=\Theta_{k-1} \cap \Delta_{k}
$$

with initial condition $\Theta_{0}=\Theta$.

Lemma 2 (Membership set) The set $\Theta_{k}$ is a convex, polytopic set explicitly given in half-space form

$$
\Theta_{k}=\left\{\theta \in \mathbb{R}^{p} \mid H_{\theta_{k}} \theta \leq h_{\theta_{k}}\right\}
$$

with $H_{\theta_{k}} \in \mathbb{R}^{q_{k} \times p}$ and $h_{\theta_{k}} \in \mathbb{R}^{q_{k}}$. Furthermore, $\theta^{*} \in \Theta_{k}$ and $\Theta_{k+1} \subseteq \Theta_{k}$.

Although not necessary from a theoretical perspective, a minimal representation of $\Theta_{k}$ should be used to decrease the computational load of the MPC algorithm. Redundant constraints in (8) can be removed efficiently by solving a series of linear programs, cf. [8, Section 3.3].
Bounded complexity set update Under the update law (7), the number of non-redundant half-spaces defining $\Theta_{k}$ in (8) could in principle grow without bound, making it necessary to use an approximation of the minimal non-falsified parameter set under which Lemma 2 remains valid. In the literature, several methods for bounded complexity set updates by means of simpler regions have been proposed, including using ellipsoids [13], parallelotopes [10] or more general polytopes [38]. All methods based on polytopic sets limit the complexity by explicitly limiting the number of halfspaces. Similar to using ellipsoids, the following update rule ensures a constant complexity of the parameter set, but is more flexible.

Let $H_{\theta} \in \mathbb{R}^{q \times p}$ be an a priori chosen matrix whose rows define $q$ normal directions of the facets of a polytope that bounds the estimated parameter set. At each time step $k$ the estimated membership set is given by $\Theta_{k}=$ $\left\{\theta \in \mathbb{R}^{p} \mid H_{\theta} \theta \leq h_{\theta_{k}}\right\}$ with $h_{\theta_{0}} \in \mathbb{R}^{q}$ satisfying $\Theta \subseteq \Theta_{0}$ and $h_{\theta_{k+1}}$ being a minimizer of

$$
\begin{aligned}
& \min _{h \in \mathbb{R}^{q}, \Lambda \in \mathbb{R}_{\geq 0}^{q \times p_{w}}} \mathbf{1}^{\top} h \\
& \text { s.t. } \Lambda\left[\begin{array}{c}
H_{\theta} \\
-H_{w} D_{k}
\end{array}\right]=H_{\theta} \\
& \Lambda\left[\begin{array}{c}
h_{\theta_{k}} \\
\mathbf{1}+H_{w} d_{k+1}
\end{array}\right] \leq h
\end{aligned}
$$

with $p_{w}=q+q_{w}$.

Lemma 3 Let $h_{\theta_{k}}$ be chosen by (9) with $h_{\theta_{0}} \in \mathbb{R}^{q}$ such that $\Theta \subseteq \Theta_{0}$, then $\theta^{*} \in \Theta_{k}$ and

$$
\Theta_{k} \cap \Delta_{k+1} \subseteq \Theta_{k+1} \subseteq \Theta_{k}
$$

PROOF. The constraints in (9) are equivalent to $\Theta_{k} \cap$ $\Delta_{k+1} \subseteq \Theta_{k+1}[21]$, which by induction implies $\theta^{*} \in$ $\Theta_{k}$ for all $k \geq 0$. Furthermore $\Lambda=\left[\begin{array}{ll}I & 0\end{array}\right], h=h_{\theta_{k}}$ is a feasible solution for (9) and since the constraints on the individual rows of $[h, \Lambda]$ are decoupled, it necessarily holds that $h^{*} \leq h_{\theta_{k}}$, which implies $\Theta_{k+1} \subseteq \Theta_{k}$.

Remark 4 As proposed in [10], instead of an update in each iteration, block processing every $n_{u}$ steps using $\Delta_{k}$, $\ldots, \Delta_{k-n_{u}}$ or a moving window could be used to achieve a tighter set estimate without changing the result.

To decrease the computational load, similar to [38] one could update only the entries $\left[h_{\theta_{k}}\right]_{i}$ of facets $i$ that are closest to facets in $\Delta_{k}$ in an inner product sense. But note that the update algorithm proposed in [38] does not always yield the best reduction in size as only one (and not necessarily the best) facet is updated. 
Point estimate Methods based on Kalman filters or Recursive Least Squares have been suggested for parameter estimation. Instead, in order to provide closed-loop stability guarantees, we propose a Least Mean Squares (LMS) filter. For FIR models, in [16], the LMS filter has been shown to be an $\mathcal{H}_{\infty}$ optimal map from the disturbance sequence to the sequence of prediction errors, reaching the best achievable gain of 1 .

Given a parameter estimate $\hat{\theta}_{k}$, denote the predicted state by $\hat{x}_{1 \mid k}=A\left(\hat{\theta}_{k}\right) x_{k}+B\left(\hat{\theta}_{k}\right) u_{k}$ and the prediction error by

$$
\tilde{x}_{1 \mid k}=A\left(\theta^{*}\right) x_{k}+B\left(\theta^{*}\right) u_{k}-\hat{x}_{1 \mid k} .
$$

For given $\hat{\theta}_{0} \in \Theta$ and parameter update gain $\mu \in \mathbb{R}_{>0}$ satisfying $\frac{1}{\mu}>\sup _{(x, u) \in \mathbb{Z}}\|D(x, u)\|^{2}$ the estimate $\hat{\theta}_{k}$ is defined recursively by

$$
\begin{aligned}
& \tilde{\theta}_{k}=\hat{\theta}_{k-1}+\mu D\left(x_{k-1}, u_{k-1}\right)^{\top}\left(x_{k}-\hat{x}_{1 \mid k-1}\right) \\
& \hat{\theta}_{k}=\Pi_{\Theta}\left(\tilde{\theta}_{k}\right)
\end{aligned}
$$

where $\Pi_{\Theta}(\tilde{\theta})=\arg \min _{\theta \in \Theta}\|\theta-\tilde{\theta}\|$ denotes the Euclidean projection of a point $\tilde{\theta} \in \mathbb{R}^{p}$ onto the set $\Theta$.

The following lemma summarizes the relevant properties, which will be used in the following stability analysis of the MPC closed loop.

Lemma 5 (Point estimate) If $\sup _{k \in \mathbb{N}}\left\|x_{k}\right\|<\infty$, $\sup _{k \in \mathbb{N}}\left\|u_{k}\right\|<\infty$, then the parameter estimate $\hat{\theta}_{k}$ is bounded, in accordance with the prior parameter set, i.e. $\hat{\theta}_{k} \in \Theta$, and

$$
\sup _{m \in \mathbb{N}, w_{k} \in \mathbb{W}, \hat{\theta}_{0} \in \Theta} \frac{\sum_{k=0}^{m}\left\|\tilde{x}_{1 \mid k}\right\|^{2}}{\frac{1}{\mu}\left\|\hat{\theta}_{0}-\theta^{*}\right\|^{2}+\sum_{k=0}^{m}\left\|w_{k}\right\|^{2}} \leq 1 .
$$

PROOF. Boundedness of $\hat{\theta}_{k}$ and $\hat{\theta}_{k} \in \Theta$ follow trivially from the set update $(6),(7)$ and projection. To prove the bound on the prediction error consider

$$
\begin{aligned}
& \frac{1}{\mu}\left\|\hat{\theta}_{k+1}-\theta^{*}\right\|^{2}-\frac{1}{\mu}\left\|\hat{\theta}_{k}-\theta^{*}\right\|^{2} \\
& \leq \frac{1}{\mu}\left\|\tilde{\theta}_{k+1}-\theta^{*}\right\|^{2}-\frac{1}{\mu}\left\|\hat{\theta}_{k}-\theta^{*}\right\|^{2} \\
& =\frac{1}{\mu}\left\|\tilde{\theta}_{k+1}-\hat{\theta}_{k}\right\|^{2}+\frac{2}{\mu}\left(\tilde{\theta}_{k+1}-\hat{\theta}_{k}\right)^{\top}\left(\hat{\theta}_{k}-\theta^{*}\right) \\
& =\frac{1}{\mu}\left\|\mu D_{k}^{\top}\left(\tilde{x}_{1 \mid k}+w_{k}\right)\right\|^{2}+2\left(\tilde{x}_{1 \mid k}+w_{k}\right)^{\top} D_{k}\left(\hat{\theta}_{k}-\theta^{*}\right) \\
& \leq\left(\mu\left\|D_{k}\right\|^{2}-1\right)\left\|\tilde{x}_{1 \mid k}+w_{k}\right\|^{2}-\left\|\tilde{x}_{1 \mid k}\right\|^{2}+\left\|w_{k}\right\|^{2} \\
& \leq-\left\|\tilde{x}_{1 \mid k}\right\|^{2}+\left\|w_{k}\right\|^{2}
\end{aligned}
$$

with $D_{k}=D\left(x_{k}, u_{k}\right)$. The first inequality follows from non-expansiveness of the projection operator and $\theta^{*} \in$ $\Theta$. In the second equality and inequality we use (12) with $x_{k+1}-\hat{x}_{1 \mid k}=\tilde{x}_{1 \mid k}+w_{k}, \tilde{x}_{1 \mid k}=D_{k}\left(\theta^{*}-\hat{\theta}_{k}\right)$, and completion of squares. Summing (13) from $k=0$ to $m$ yields

$\frac{1}{\mu}\left\|\hat{\theta}_{m+1}-\theta^{*}\right\|^{2}+\sum_{k=0}^{m}\left\|\tilde{x}_{1 \mid k}\right\|^{2} \leq \sum_{k=0}^{m}\left\|w_{k}\right\|^{2}+\frac{1}{\mu}\left\|\hat{\theta}_{0}-\theta^{*}\right\|^{2}$,

which proves the claim.

Corollary 6 If $\sup _{k \in \mathbb{N}}\left\|x_{k}\right\|<\infty, \sup _{k \in \mathbb{N}}\left\|u_{k}\right\|<\infty$, and $\sum_{k=0}^{\infty}\left\|w_{k}\right\|^{2}<\infty$, then the prediction error converges to 0 asymptotically, i.e., $\lim _{k \rightarrow \infty}\left\|\tilde{x}_{1 \mid k}\right\|=0$.

Remark 7 In order to speed up parameter convergence, in (12) a projection onto the set $\Theta_{k}$ could be used without changing the result in Lemma 5. Projecting the parameter estimate onto a trust region is common practice for robust adaptive control algorithms [32]. Furthermore, note that the update gain $\mu$ is non-increasing with an increasing size of $\mathbb{Z}$, which is in line with common recommendations on stabilizing indirect adaptive control, c.f. [7, Chapter 7.4].

\subsection{State-tube and constraint satisfaction}

To cope with state predictions under uncertainty an input parametrization

$$
u_{l \mid k}(x)=K x+v_{l \mid k}
$$

is introduced with decision variables $\mathbf{v}_{N \mid k}=\left\{v_{l \mid k}\right\}_{l \in \mathbb{N}_{0}^{N-1}}$, $v_{l \mid k} \in \mathbb{R}^{m}$ and prestabilizing feedback gain $K \in \mathbb{R}^{m \times n}$ satisfying the following assumption.

Assumption 8 (Prestabilization) The feedback gain $K$ is chosen such that $A_{c l}(\theta)=A(\theta)+B(\theta) K$ is stable for all $\theta \in \Theta$.

While this assumption, compared to most adaptive control literature, is conservative, it is necessary to prove robust constraint satisfaction. Note that, given the initial parameter set $\Theta$, the gain $K$ can be determined by standard robust control methods.

By restricting the sets $\mathbb{X}_{l \mid k}$ to be translations and dilations of a given polytope $\mathbb{X}_{0}$, the MPC optimization can be recast as a computationally tractable, finite dimensional optimization program. In the context of robust $\mathrm{MPC}$, the following parametrization has previously been introduced in [22] and [30].

For a given polytope $\mathbb{X}_{0}=\left\{x \in \mathbb{R}^{n} \mid H_{x} x \leq \mathbf{1}\right\}$ with vertices $\left\{x^{1}, \ldots, x^{v}\right\}$, MPC decision variables $\mathbf{z}_{N \mid k}=$ 
$\left\{z_{l \mid k}\right\}_{l \in \mathbb{N}_{0}^{N}}, z_{l \mid k} \in \mathbb{R}^{n}$, and $\boldsymbol{\alpha}_{N \mid k}=\left\{\alpha_{l \mid k}\right\}_{l \in \mathbb{N}_{0}^{N}}, \alpha_{l \mid k} \in$ $\mathbb{R}_{\geq 0}$, define

$$
\begin{aligned}
\mathbb{X}_{l \mid k} & =\left\{z_{l \mid k}\right\} \oplus \alpha_{l \mid k} \mathbb{X}_{0} \\
& =\left\{x \in \mathbb{R}^{n} \mid H_{x}\left(x-z_{l \mid k}\right) \leq \alpha_{l \mid k} \mathbf{1}\right\} \\
& =\left\{z_{l \mid k}\right\} \oplus \alpha_{l \mid k} \operatorname{co}\left\{x^{1}, x^{2}, \ldots, x^{v}\right\} .
\end{aligned}
$$

The reason for choosing this tube parameterization is the explicit description of $\mathbb{X}_{l \mid k}$ in both vertex and half-space form. This can be exploited to reformulate the prediction constraints (5) as linear constraints while taking into account the online updated parameter set $\Theta_{k}$.

For a more compact notation in the following proposition, define $d_{l \mid k}^{j}=A_{0} x_{l \mid k}^{j}+B_{0} u_{l \mid k}^{j}-z_{l+1 \mid k}$ and $D_{l \mid k}^{j}=D\left(x_{l \mid k}^{j}, u_{l \mid k}^{j}\right)$, where $x_{l \mid k}^{j}=z_{l \mid k}+\alpha_{l \mid k} x^{j}$ and $u_{l \mid k}^{j}=u_{l \mid k}\left(x_{l \mid k}^{j}\right)$. Furthermore, for all $i \in \mathbb{N}_{1}^{u}$ define $[\bar{w}]_{i}=\max _{w \in \mathbb{W}}\left[H_{x}\right]_{i} w$ and for all $i \in \mathbb{N}_{1}^{c}$ define $[\bar{f}]_{i}=\max _{x \in \mathbb{X}_{0}}[F+G K]_{i} x$ with $F, G$ from (4).

Proposition 9 (Prediction tube) Let $\left\{\mathbb{X}_{l \mid k}\right\}_{l \in \mathbb{N}_{0}^{N}}$ be parametrized as in (14) with decision variables $\mathbf{z}_{N \mid k}$, $\boldsymbol{\alpha}_{N \mid k}$, and $\mathbf{v}_{N \mid k}$.

Equations (5a)-(5c) are satisfied if and only if for all $j \in \mathbb{N}_{1}^{v}, l \in \mathbb{N}_{0}^{N-1}$ there exists $\Lambda_{l \mid k}^{j} \in \mathbb{R}_{\geq 0}^{u \times q_{k}}$ such that

$$
\begin{aligned}
& (F+G K) z_{l \mid k}+G v_{l \mid k}+\alpha_{l \mid k} \bar{f} \leq \mathbf{1} \\
& -H_{x} z_{0 \mid k}-\alpha_{0 \mid k} \mathbf{1} \leq-H_{x} x_{k} \\
& \Lambda_{l \mid k}^{j} h_{\theta_{k}}+H_{x} d_{l \mid k}^{j}-\alpha_{l+1 \mid k} \mathbf{1} \leq-\bar{w} \\
& H_{x} D_{l \mid k}^{j}=\Lambda_{l \mid k}^{j} H_{\theta_{k}} .
\end{aligned}
$$

The value of Proposition 9 is that it allows the constraints on state tubes to be incorporated as linear constraints into the MPC optimization.

PROOF. Inequality (5c) is equivalent to

$(F+G K) z_{l \mid k}+G v_{l \mid k}+\alpha_{l \mid k}(F+G K) x \leq \mathbf{1} \quad \forall x \in \mathbb{X}_{0}$,

which is equivalent to (15a) when maximized over $x \in$ $\mathbb{X}_{0}$.

Inequality (5a) is equivalent to (15b), and (5b) is equivalent to $(15 \mathrm{c}),(15 \mathrm{~d})$ as shown by the following reformu- lation.

$$
\begin{aligned}
& \mathbb{X}_{l+1 \mid k} \supseteq A_{c l}(\theta) \mathbb{X}_{l \mid k} \oplus B(\theta) v_{l \mid k} \oplus \mathbb{W} \quad \forall \theta \in \Theta_{k} \\
& \Leftrightarrow H_{x}\left(A_{c l}(\theta) x+B(\theta) v_{l \mid k}+w-z_{l+1 \mid k}\right) \leq \alpha_{l+1 \mid k} \mathbf{1} \\
& \forall x \in \mathbb{X}_{l \mid k}, \theta \in \Theta_{k}, w \in \mathbb{W} \\
& \Leftrightarrow H_{x}\left(A_{c l}(\theta)\left(z_{l \mid k}+\alpha_{l \mid k} x^{j}\right)+B(\theta) v_{l \mid k}-z_{l+1 \mid k}\right) \\
& -\alpha_{l+1 \mid k} \mathbf{1} \leq-\bar{w} \quad \forall j \in \mathbb{N}_{1}^{v}, \theta \in \Theta_{k} \\
& \Leftrightarrow \max _{\theta \in \Theta_{k}}\left\{H_{x}\left(A_{c l}(\theta)\left(z_{l \mid k}+\alpha_{l \mid k} x^{j}\right)+B(\theta) v_{l \mid k}\right)\right\} \\
& -H_{x} z_{l+1 \mid k}-\alpha_{l+1 \mid k} \mathbf{1} \leq-\bar{w} \quad \forall j \in \mathbb{N}_{1}^{v} \\
& \Leftrightarrow \max _{\theta \in \Theta_{k}}\left\{H_{x} D_{l \mid k}^{j} \theta\right\}+H_{x} d_{l \mid k}^{j}-\alpha_{l+1 \mid k} \mathbf{1} \leq-\bar{w} \quad \forall j \in \mathbb{N}_{1}^{v} \\
& \Leftrightarrow\left\{\begin{array}{l}
\Lambda_{l \mid k}^{j} h_{\theta_{k}}+H_{x} d_{l \mid k}^{j}-\alpha_{l+1 \mid k} \mathbf{1} \leq-\bar{w} \\
H_{x} D_{l \mid k}^{j}=\Lambda_{l \mid k}^{j} H_{\theta_{k}} \\
\Lambda_{l \mid k}^{j} \in \mathbb{R}_{\geq 0}^{u \times q_{k}}
\end{array}\right\} \quad \forall j \in \mathbb{N}_{1}^{v}
\end{aligned}
$$

The first equivalence follows from the equivalence of $x \in$ $\mathbb{X}_{l+1 \mid k}$ with $H_{x}\left(x-z_{l+1 \mid k}\right) \leq \alpha_{l+1 \mid k} 1$. The second follows from the left hand side being convex in $x$ for given $\theta$ so that therefore the inequality holds for all $x \in \mathbb{X}_{l \mid k}$ if and only if it holds for the vertices of $\mathbb{X}_{l \mid k}$. In the third equivalence the maximisation is to be understood rowwise and the last equivalence follows from strong duality in linear programming, cf. [6]. Specifically, considering row $i \in \mathbb{N}_{1}^{u}$ in the maximisation, which is finite since $\Theta_{k}$ is compact, we have that

$$
\begin{aligned}
& \max _{\theta \in \Theta_{k}}\left\{\left[H_{x}\right]_{i} D_{l \mid k}^{j} \theta\right\} \\
= & \min _{\lambda_{i} \in \mathbb{R}_{\geq 0}^{q_{k}}} \max _{\theta}\left\{\left[H_{x}\right]_{i} D_{l \mid k}^{j} \theta+\lambda_{i}^{\top}\left(h_{\theta_{k}}-H_{\theta_{k}} \theta\right)\right\} \\
= & \min _{\lambda_{i} \in \mathbb{R}_{\geq 0}^{q_{k}}} \lambda_{i}^{\top} h_{\theta_{k}} \\
& \text { s.t. }\left[H_{x}\right]_{i} D_{l \mid k}^{j}-\lambda_{i}^{\top} H_{\theta_{k}}=0 .
\end{aligned}
$$

Since the minimization in the inequality constraint can be removed, this concludes the proof.

Remark 10 The equality constraint (15d) can equivalently be written in vector form as a linear constraint

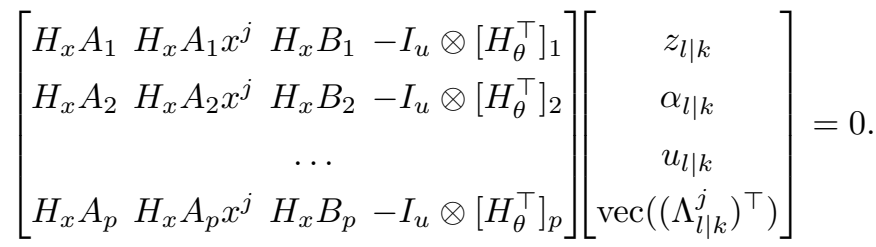

Lemma 2 and Proposition 9 constitute the basic framework for a predictive control algorithm with model adaption and robust constraint satisfaction. In the next section, this is complemented with a suitable terminal constraint and a convex, positive definite objective function to derive a stabilizing, adaptive MPC scheme. 


\subsection{Terminal constraint and objective function}

As is common in stabilizing MPC, to prove stability and recursive feasibility, we assume the existence of a robustly invariant terminal set for the state tube. Note that if the system dynamics are stable and no state constraints are present, the terminal constraint can be omitted by setting the prestabilizing feedback gain $K$ to zero.

Assumption 11 (Terminal set) There exists a nonempty terminal set $\mathbb{X}_{f}=\left\{(z, \alpha) \in \mathbb{R}^{n} \times \mathbb{R}_{\geq 0} \mid H_{T} z+\right.$ $\left.h_{T} \alpha \leq \mathbf{1}\right\}$ such that $(x, K x) \in \mathbb{Z}$ for all $x \in\{z\} \oplus \alpha \mathbb{X}_{0}$, $(z, \alpha) \in \mathbb{X}_{f}$ and for all $\theta \in \Theta$ we have

$$
\begin{aligned}
(z, \alpha) \in \mathbb{X}_{f} & \Longrightarrow \exists\left(z^{+}, \alpha^{+}\right) \in \mathbb{X}_{f} \text { s.t. } \\
& A_{c l}(\theta)\left(\{z\} \oplus \alpha \mathbb{X}_{0}\right) \oplus \mathbb{W} \subseteq\left\{z^{+}\right\} \oplus \alpha^{+} \mathbb{X}_{0} .
\end{aligned}
$$

The assumption on the terminal set $\mathbb{X}_{f}$ is analogous to the terminal set in robust MPC with homothetic tubes, cf. [29]. It can be computed recursively, following standard algorithms to determine a robust invariant set through considering the dynamics of $(z, \alpha)$. For completeness, an explicit algorithm is given in Appendix A.1.

In the following, we use the point estimate $\hat{\theta}_{k}$ to define a certainty equivalence cost, which leads to a finite $\ell_{2}$ gain from the disturbance to the state of the closed-loop system. Similar to nominal MPC for linear systems, a quadratic cost on the nominal predicted state and input based on the parameter estimate $\hat{\theta}_{k}$ is employed. Let $Q \in \mathbb{R}^{n \times n}$ and $R \in \mathbb{R}^{m \times m}$ be positive definite cost matrices and let $P \in \mathbb{R}^{n \times n}$ satisfy

$$
A_{c l}(\theta)^{\top} P A_{c l}(\theta)+Q+K^{\top} R K \preceq P \quad \forall \theta \in \Theta .
$$

The finite horizon MPC cost function is then given by

$$
J_{N}\left(x_{k}, \hat{\theta}_{k}, \mathbf{v}_{N \mid k}\right)=\sum_{l=0}^{N-1}\left\|\hat{x}_{l \mid k}\right\|_{Q}^{2}+\left\|\hat{u}_{l \mid k}\right\|_{R}^{2}+\left\|\hat{x}_{N \mid k}\right\|_{P}^{2}
$$

where $\hat{x}_{l \mid k}, \hat{u}_{l \mid k}$ are defined recursively by

$$
\begin{aligned}
& \hat{x}_{l+1 \mid k}=A\left(\hat{\theta}_{k}\right) \hat{x}_{l \mid k}+B\left(\hat{\theta}_{k}\right) \hat{u}_{l \mid k}, \quad x_{0 \mid k}=x_{k}, \\
& \hat{u}_{l \mid k}=K \hat{x}_{l \mid k}+v_{l \mid k} .
\end{aligned}
$$

Remark 12 Note that in the proposed cost function it is not necessary to use "delayed" parameter estimates as proposed in [11], where the updated parameter is inserted only at the end of the prediction horizon. Delayed parameter estimates allow for a simpler analysis but slow down the convergence of the prediction error.

Remark 13 Alternatively, as proposed in [23], based only on the set estimate $\Theta_{k}$, a min-max cost

$$
\ell\left(z_{l \mid k}, \alpha_{l \mid k}, v_{l \mid k}\right)=\max _{x \in \mathbb{X}_{l \mid k}}\left\{\|Q x\|_{\infty}+\left\|R u_{l \mid k}(x)\right\|_{\infty}\right\}
$$

can be employed, which has the advantage that the point estimate $\hat{\theta}_{k}$ for the parameter can be omitted. With a suitable terminal cost, and under an additional, technical assumption, practical stability of the closed loop has been proved. Yet, the disadvantage of the approach in [23] is that the ultimate bound on the state depends on the set $\mathbb{W}$ whereas the approach proposed in this paper, i.e. using the point estimate $\theta_{k}$, allows the $\ell_{2}$ norm or the state to be bounded in terms of the $\ell_{2}$ norm of the realized disturbance sequence.

\subsection{MPC algorithm and stability analysis}

Having derived tractable reformulations for the parameter estimation and optimal control problem, we summarize the adaptive MPC algorithm and provide a brief analysis of the relevant control theoretic properties.

In order to simplify notation, we denote the decision variables in the online optimization program by $\mathbf{d}_{N \mid k}=\left\{\mathbf{z}_{N \mid k}, \boldsymbol{\alpha}_{N \mid k}, \mathbf{v}_{N \mid k}, \boldsymbol{\Lambda}_{N \mid k}\right\}$ with $\boldsymbol{\Lambda}_{N \mid k}=$

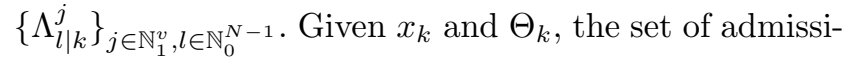
ble decision variables is

$$
\mathbb{D}\left(x_{k}, \Theta_{k}\right)=\left\{\mathbf{d}_{N \mid k} \mid(15),\left(z_{N \mid k}, \alpha_{N \mid k}\right) \in \mathbb{X}_{f}\right\}
$$

The adaptive MPC algorithm can be summarized as follows.

Offline: Choose cost matrices $Q$ and $R$. Determine a robustly stabilizing feedback gain $K$, terminal set $\mathbb{X}_{f}$ according to Assumption 11, and terminal cost matrix $P$ satisfying (16).

Online: For each time step $k=0,1,2, \ldots$

(i) Measure the state $x_{k}$.

(ii) If $k>0$ update the membership set $\Theta_{k}$ and point estimate $\hat{\theta}_{k}$ according to (7) or (9), and (12), respectively.

(iii) Determine the minimizer of the linearly constrained quadratic program

$$
\begin{array}{r}
\mathbf{d}_{N \mid k}^{*}=\arg \min _{\mathbf{d}_{N \mid k}} J_{N}\left(x_{k}, \hat{\theta}_{k}, \mathbf{v}_{N \mid k}\right) \\
\text { s.t. } \mathbf{d}_{N \mid k} \in \mathbb{D}\left(x_{k}, \Theta_{k}\right) .
\end{array}
$$

(iv) Apply

$$
u_{k}=K x_{k}+v_{0 \mid k}^{*}
$$

The following theorem establishes recursive feasibility of the adaptive MPC algorithm, consistent parameter estimation, robust satisfaction of the constraints (4), and 
finite gain $\ell_{2}$ stability of the closed loop. Recursive feasibility guarantees a well-defined control law; if there exists a solution to the optimization program for a given initial condition, a solution to the optimization program exists for all future states resulting from the application of the proposed MPC control law. Consistent parameter estimation implies that the true parameter is always contained in the estimated parameter set and the estimated parameter is consistent with the initially given parameter set.

Theorem 14 (Closed-loop properties) Suppose Assumptions 1 and 11 are satisfied. If $\theta^{*} \in \Theta_{k_{0}}$, $\mathbb{D}\left(x_{k_{0}}, \Theta_{k_{0}}\right) \neq \emptyset$, then for all $k \geq k_{0}$

(i) $\mathbb{D}\left(x_{k}, \Theta_{k}\right) \neq \emptyset$

(ii) $\theta^{*} \in \Theta_{k}, \hat{\theta}_{k} \in \Theta$

(iii) $x_{k} \times u_{k} \in \mathbb{Z}$.

Moreover, the system (1) in closed loop with the proposed $M P C$ control law $u\left(x_{k}\right)=K x_{k}+v_{0 \mid k}^{*}$ is finite gain $\ell_{2}$ stable, i.e., there exist constants $c_{0}, c_{1}, c_{2} \in \mathbb{R}_{>0}$ such that for all $K \in \mathbb{N}$

$$
\sum_{k=0}^{K}\left\|x_{k}\right\|^{2} \leq c_{0}\left\|x_{0}\right\|^{2}+c_{1}\left\|\hat{\theta}_{0}-\theta^{*}\right\|^{2}+c_{2} \sum_{k=0}^{K}\left\|w_{k}\right\|^{2}
$$

Remark 15 Note that, unlike nominal MPC, the controller is not a static state feedback, but a dynamic controller with states $\hat{\theta}_{k}$ and $\Theta_{k}$. The term $c_{0}\left\|x_{0}\right\|^{2}+c_{1} \| \hat{\theta}_{0}-$ $\theta^{*} \|^{2}$ bounds the possible overshoot due to the initial condition of the plant and the controller.

PROOF. We prove claim (i) and (ii) for $k=k_{0}+1$; since $k_{0}$ is arbitrary the results follow by induction.

Claim (i): At time $k_{0}$, for $l \in \mathbb{N}_{0}^{N-1}$, let $u_{l \mid k_{0}}(x)=$ $K x+v_{l \mid k_{0}}^{*}$ and $\mathbb{X}_{l+1 \mid k_{0}}^{*}$ be a feasible input and admissible state tube trajectory satisfying the MPC constraints (5) and terminal constraint $\mathbb{X}_{N \mid k_{0}} \in \mathbb{X}_{f}$. For time $k_{0}+1$ and $l \in \mathbb{N}_{0}^{N-1}$ define the candidate input $\tilde{u}_{l \mid k_{0}+1}(x)=K x+v_{l+1 \mid k_{0}}^{*}$ with $v_{N \mid k_{0}}^{*}=0$ and candidate state tube $\tilde{\mathbb{X}}_{l \mid k_{0}+1}=\mathbb{X}_{l+1 \mid k_{0}}^{*}$. Since $\tilde{\mathbb{X}}_{N-1 \mid k_{0}+1} \subseteq \mathbb{X}_{f}$ and $\Theta_{k_{0}} \subseteq \Theta$, by Assumption 11, there exists $\tilde{\mathbb{X}}_{N \mid k_{0}+1} \subseteq$ $\mathbb{X}_{f}$ satisfying $A(\theta) x \oplus B(\theta) \tilde{u}_{N-1 \mid k_{0}+1}(x) \oplus \mathbb{W} \subseteq$ $\tilde{\mathbb{X}}_{N \mid k_{0}+1}$ for all $x \in \tilde{\mathbb{X}}_{N-1 \mid k_{0}+1}, \theta \in \Theta_{k_{0}}$. By construction $\left\{\tilde{u}_{l \mid k_{0}+1}, \quad \tilde{\mathbb{X}}_{l \mid k_{0}+1}\right\}_{l \in \mathbb{N}_{0}^{N-1}}$ satisfy the constraints (5b), (5c), and since $x_{k_{0}+1}=A\left(\theta^{*}\right) x_{k_{0}}+$ $B\left(\theta^{*}\right) u_{k_{0}}+w_{k_{0}} \in \mathbb{X}_{1 \mid k_{0}}^{*}=\tilde{\mathbb{X}}_{0 \mid k_{0}+1}$, constraint (5a) is satisfied. By Proposition 9 this is equivalent to feasibility of (15) and hence $\mathbb{D}\left(x_{k_{0}+1}, \Theta_{k_{0}}\right) \neq \emptyset$, which implies $\mathbb{D}\left(x_{k_{0}+1}, \Theta_{k_{0}+1}\right) \neq \emptyset$ as $\Theta_{k_{0}+1} \subseteq \Theta_{k_{0}}$.
Claim (ii): If $x_{k_{0}}$ is bounded, the optimal solution $v_{0 \mid k_{0}}^{*}$ and hence $u_{k_{0}}$ and $x_{k_{0}+1}$ are well defined and bounded [33]. The claim then follows from Lemma 2 and 5 .

Claim (iii) is a direct corollary of $\mathbb{D}\left(x_{k}, \Theta_{k}\right) \neq \emptyset$ and Proposition 9.

To prove the finite $\ell_{2}$ gain note that $v_{l \mid k+1}=v_{l+1 \mid k}^{*}$ for $l \in \mathbb{N}_{0}^{N-2}$ and $v_{N-1 \mid k+1}=0$ denotes a feasible input sequence at time $k+1$. Let $\left\{\hat{x}_{l \mid k}\right\}_{l \in \mathbb{N}_{1}^{N}}$ and $\left\{\hat{x}_{l-1 \mid k+1}\right\}_{l \in \mathbb{N}_{1}^{N}}$ denote the corresponding predicted state trajectories, which evolve according to (18) with initial conditions $x_{k}$ and $x_{k+1}$, respectively, and denote the difference by

$$
\begin{aligned}
\delta \hat{x}_{l \mid k}= & \hat{x}_{l-1 \mid k+1}-\hat{x}_{l \mid k} \\
= & A_{c l}\left(\hat{\theta}_{k+1}\right)^{l-1}\left(w_{k}+\tilde{x}_{1 \mid k}\right) \\
& \quad+\sum_{i=1}^{l-1} A_{c l}\left(\hat{\theta}_{k+1}\right)^{l-1-i} D\left(\hat{x}_{i \mid k}, \hat{u}_{i \mid k}\right)\left(\hat{\theta}_{k+1}-\hat{\theta}_{k}\right) .
\end{aligned}
$$

By non-expansiveness of the projection operator, we have $\left\|\hat{\theta}_{k+1}-\hat{\theta}_{k}\right\| \leq\left\|\tilde{\theta}_{k+1}-\hat{\theta}_{k}\right\|$, and using (12) together with $\frac{1}{\mu}>\sup _{(x, u) \in \mathbb{Z}}\|D(x, u)\|^{2}$ this leads to

$$
\left\|\delta \hat{x}_{l \mid k}\right\| \leq\left(\sum_{i=0}^{l-1}\left\|A_{c l}\left(\hat{\theta}_{k+1}\right)^{l-i}\right\|\right)\left\|\tilde{x}_{1 \mid k}+w_{k}\right\| .
$$

The claim then follows by a standard argument. With $\bar{Q}=Q+K^{\top} R K$ consider

$$
\begin{aligned}
& V_{N}\left(x_{k+1}, \hat{\theta}_{k+1}, \Theta_{k+1}\right)-V_{N}\left(x_{k}, \hat{\theta}_{k}, \Theta_{k}\right) \\
\leq & J_{N}\left(x_{k+1}, \hat{\theta}_{k+1}, \tilde{\mathbf{v}}_{N \mid k}\right)-V_{N}\left(x_{k}, \hat{\theta}_{k}, \Theta_{k}\right) \\
\leq & -\left\|x_{k}\right\|_{Q}^{2}-\left\|u_{k}\right\|_{R}^{2}+\sum_{l=0}^{N-2}\left\|\hat{x}_{l \mid k+1}\right\|_{Q}^{2}+\left\|\hat{u}_{l \mid k+1}\right\|_{R}^{2} \\
& +\left\|\hat{x}_{N-1 \mid k+1}\right\|_{P}^{2}-\left(\sum_{l=1}^{N-1}\left\|\hat{x}_{l \mid k}\right\|_{Q}^{2}+\left\|\hat{u}_{l \mid k}\right\|_{R}^{2}+\left\|\hat{x}_{N \mid k}\right\|_{P}^{2}\right) \\
\leq & -\left\|x_{k}\right\|_{Q}^{2}-\left\|u_{k}\right\|_{R}^{2}+\sum_{l=1}^{N-1} \varepsilon\left(\left\|\hat{x}_{l \mid k}\right\|_{Q}^{2}+\left\|\hat{u}_{l \mid k}\right\|_{R}^{2}\right) \\
& +\varepsilon\left\|\hat{x}_{N \mid k}\right\|_{P}^{2}+\sum_{l=1}^{N-1}\left(1+\frac{1}{\varepsilon}\right)\left\|\delta \hat{x}_{l \mid k}\right\|_{\bar{Q}}^{2}+\left\|\delta \hat{x}_{N \mid k}\right\|_{P}^{2} \\
\leq & -\left\|x_{k}\right\|_{Q}^{2}+\varepsilon V_{N}\left(x_{k}, \hat{\theta}_{k}, \Theta_{k}\right)+c_{A}\left\|\tilde{x}_{1 \mid k}+w_{k}\right\|^{2} \\
\leq & -c\left\|x_{k}\right\|^{2}+c_{A}\left\|\tilde{x}_{1 \mid k}+w_{k}\right\|^{2} .
\end{aligned}
$$

The third inequality follows by Cauchy-Schwarz and Young's inequality, which implies $\left\|\hat{x}_{l-1 \mid k+1}\right\|_{Q}^{2}=\| \hat{x}_{l \mid k}+$ $\delta \hat{x}_{l \mid k}\left\|_{Q}^{2} \leq(1+\varepsilon)\right\| \hat{x}_{l \mid k}\left\|_{Q}^{2}+\left(1+\frac{1}{\varepsilon}\right)\right\| \delta x_{l \mid k} \|_{Q}^{2}$. Since for each parameter set $\Theta$ and $\theta \in \Theta, V_{N}(x, \theta, \Theta)$ is a continuous, 
piecewise quadratic function in $x$, cf. [5], it can be upper bounded by a quadratic function on the feasible set. In particular for each $\theta \in \Theta$, there exists $c_{\theta}$, such that $V_{N}(x, \theta, \Theta)=J_{N}\left(x, \theta, \mathbf{v}_{N}^{*}(x, \theta, \Theta)\right)<c_{\theta}\|x\|^{2}$, where $\mathbf{v}_{N}^{*}$ is the optimal solution of (20) with $x_{k}=x, \hat{\theta}_{k}=\theta$, $\Theta_{k}=\Theta$. Since $J_{N}\left(x, \cdot, \mathbf{v}_{N}^{*}(x, \theta, \Theta)\right)$ is continuous, we can choose an $\varepsilon>0$ such that $J_{N}\left(x, \tilde{\theta}, \mathbf{v}_{N}^{*}(x, \theta, \Theta)\right) \leq$ $c_{\theta}\|x\|^{2}$ for each $\tilde{\theta} \in \mathbb{B}_{\varepsilon}(\theta)$ and each $\theta \in \Theta$. By compactness of $\Theta$, there exists a finite collection $\left\{\theta^{i}\right\}_{i \in \mathcal{I}}$ with $\theta^{i} \in$ $\Theta$ such that $\cup_{i \in \mathcal{I}} \mathbb{B}_{\varepsilon}\left(\theta^{i}\right) \supseteq \Theta$. Hence, $J_{N}\left(x_{k}, \hat{\theta}_{k}, \mathbf{v}_{N \mid k}^{*}\right) \leq$ $\hat{c}_{\theta}\|x\|^{2}$ with $\hat{c}_{\theta}=\max _{i \in \mathcal{I}} c_{\theta^{i}}$. Thus there exist suitable constants $\varepsilon, c, c_{A} \in \mathbb{R}_{>0}$ such that the last two inequalities hold. The final result follows by summing over $k$ and using Lemma 5 and again Young's inequality for $\left\|\tilde{x}_{1 \mid k}+w_{k}\right\|^{2}$.

Corollary 16 Suppose Assumptions 1 and 11 are satisfied and $\mathbb{D}\left(x_{0}, \Theta_{0}\right) \neq \emptyset$. If $\sum_{k=0}^{\infty}\left\|w_{k}\right\|^{2}<\infty$, then $\lim _{k \rightarrow \infty} x_{k}=0$ for the system (1) in closed loop with the proposed MPC control law $u\left(x_{k}\right)=K x_{k}+v_{0 \mid k}^{*}$.

We have proved robust constraint satisfaction and stability for a computationally tractable MPC algorithm with online parameter adaption.

\section{Extensions}

Being based on a modern state-space formulation, the proposed MPC scheme with parameter adaption can be directly combined with recent results in the MPC literature, e.g. output feedback or offset-free tracking. In the following, we present two relevant modifications of the basic algorithm, one for time-varying parameters and one for persistently exciting regressors to achieve parameter convergence.

\subsection{Time-varying parameters}

We extend the admissible prediction tube and constraint reformulation to include time-varying parameters $\theta_{k}^{*}$, i.e., to include time-varying systems

$$
x_{k+1}=A\left(\theta_{k}^{*}\right) x_{k}+B\left(\theta_{k}^{*}\right) u_{k}+w_{k}
$$

under the following assumption.

\section{Assumption 17 (Time-varying parameters)}

There exists $d_{\theta} \in \mathbb{R}$, such that the parameter vector $\theta_{k}^{*}$ satisfies $\theta_{k}^{*} \in \Theta$ for all $k \in \mathbb{N}$ and

$$
\left\|\theta_{k+1}^{*}-\theta_{k}^{*}\right\| \leq d_{\theta}
$$

Assumption 17 restricts the parameter variation between consecutive time steps. Furthermore, as before, an absolute prior bound on the parameter vector is assumed, which is necessary in order to be able to guarantee robust (state) constraint satisfaction. Note that different rates of change of the parameters can easily be incorporated by a weighting matrix or a suitable scaling of the model.

To incorporate the dynamics of $\theta_{k}^{*}$, the parameter set update (7) needs to be changed. To this end, we introduce a dilation operator $\mathcal{D}_{l}$, which is defined for $l \in \mathbb{N}$, $\mathrm{d}_{\theta} \in \mathbb{R}_{>0}$, and a polytope $\Theta=\left\{\theta \in \mathbb{R}^{p} \mid H_{\theta} \theta \leq h_{\theta}\right\}$ by

$$
\mathcal{D}_{l}(\Theta)=\left\{\theta \in \mathbb{R}^{p} \mid H_{\theta} \theta \leq h_{\theta}+l d_{\theta} \mathbf{1}\right\}
$$

Using $\mathcal{D}_{l}$ and assuming (without loss of generality) $\left\|\left[H_{\theta_{k}}\right]_{i}\right\|=1$ for all $i \in \mathbb{N}_{1}^{q_{k}}$, the online parameter set estimation (7) becomes

$$
\Theta_{k}=\mathcal{D}_{1}\left(\Theta_{k-1} \cap \Delta_{k}\right) \cap \Theta .
$$

Similarly, to ensure constraint satisfaction, the equation for admissible state tubes (5b) needs to be adapted. Specifically, the open-loop prediction for the estimated parameter membership set needs to be changed from $\Theta_{k}$ to

$$
\Theta_{l \mid k}=\mathcal{D}_{l}\left(\Theta_{k}\right) \cap \Theta .
$$

Note that $\mathcal{D}_{l}$ is easily implemented for sets that are given in half space form as only the right hand side needs to be changed. Thus, analogous to Proposition 9, the admissible prediction tube for systems with time-varying parameters, including the parameter set prediction (26), can be reformulated as linear constraints.

Proposition 18 (Prediction tube) Let $\left\{\mathbb{X}_{l \mid k}\right\}_{l \in \mathbb{N}_{0}^{N}}$ be parametrized as in (14) with decision variables $\mathbf{z}_{N \mid k}$, $\boldsymbol{\alpha}_{N \mid k}$, and $\mathbf{v}_{N \mid k}$. Without loss of generality, assume $\left\|\left[H_{\theta}\right]_{i}\right\|=1,\left\|\left[H_{\theta_{k}}\right]_{i}\right\|=1$ for all $i \in \mathbb{N}_{1}^{q_{k}}$.

The prediction tube equations

$$
\begin{aligned}
& \mathbb{X}_{0 \mid k} \ni x_{k}, \\
& \mathbb{X}_{l+1 \mid k} \supseteq A_{c l}(\theta) \mathbb{X}_{l \mid k} \oplus B(\theta) v_{l \mid k} \oplus \mathbb{W} \quad \forall \theta \in \Theta_{l+k}
\end{aligned}
$$

are satisfied if, for all $j \in \mathbb{N}_{1}^{v}, l \in \mathbb{N}_{0}^{N-1}$, there exists $\Lambda_{l \mid k}^{j} \in \mathbb{R}_{\geq 0}^{u \times q_{k}}$ such that

$$
\begin{aligned}
& -H_{x} z_{0 \mid k}-\alpha_{0 \mid k} \mathbf{1} \leq-H_{x} x_{k} \\
& \Lambda_{l \mid k}^{j} h_{\theta_{l \mid k}}+H_{x} d_{l \mid k}^{j}-\alpha_{l+1 \mid k} \mathbf{1} \leq-\bar{w} \\
& H_{x} D_{l \mid k}^{j}=\Lambda_{l \mid k}^{j} H_{\theta_{l \mid k}}
\end{aligned}
$$

with

$H_{\theta_{l \mid k}}=\left[\begin{array}{c}H_{\theta_{k}} \\ H_{\theta}\end{array}\right] \in \mathbb{R}^{q_{k}^{\prime} \times p}, \quad h_{\theta_{l \mid k}}=\left[\begin{array}{c}h_{\theta_{k}}+l d_{\theta} \mathbf{1} \\ h_{\theta}\end{array}\right] \in \mathbb{R}^{q_{k}^{\prime}}$ 
PROOF. The proof follows analogously to the case with non time-varying parameters and by noting that $\left\{\theta \in \mathbb{R}^{p} \mid H_{\theta_{l \mid k}} \theta \leq h_{\theta_{l \mid k}}\right\}=\mathcal{D}_{l}\left(\Theta_{k}\right) \cap \Theta=\Theta_{l \mid k} \subseteq \Theta_{l+k}$.

$$
\begin{aligned}
& \mathbb{X}_{l+1 \mid k} \supseteq A_{c l}(\theta) \mathbb{X}_{l \mid k} \oplus B(\theta) v_{l \mid k} \oplus \mathbb{W} \\
& \Leftarrow \mathbb{X}_{l+1 \mid k} \supseteq A_{c l}(\theta) \mathbb{X}_{l \mid k} \oplus B(\theta) v_{l \mid k} \oplus \mathbb{W} \\
& \Leftrightarrow H_{x}\left(A_{c l}(\theta)\left(z_{l \mid k}+\alpha_{l \mid k} x^{j}\right)+B(\theta) v_{l \mid k}-z_{l+1 \mid k}\right) \\
& -\alpha_{l+1 \mid k} \mathbf{1} \leq-\bar{w} \quad \forall j \in \mathbb{N}_{1}^{v}, \theta \in \Theta_{l \mid k} \\
& \Leftrightarrow \max _{\theta \in \Theta_{l \mid k}}\left\{H_{x} D_{l \mid k}^{j} \theta\right\}+H_{x} d_{l \mid k}^{j}-\alpha_{l+1 \mid k} \mathbf{1} \leq-\bar{w} \quad \forall j \in \mathbb{N}_{1}^{v} \\
& \Leftrightarrow\left\{\begin{array}{l}
\Lambda_{l \mid k}^{j} h_{\theta_{l \mid k}}+H_{x} d_{l \mid k}^{j}-\alpha_{l+1 \mid k} \mathbf{1} \leq-\bar{w} \\
H_{x} D_{l \mid k}^{j}=\Lambda_{l \mid k}^{j} H_{\theta_{l \mid k}} \\
\Lambda_{l \mid k}^{j} \in \mathbb{R}_{\geq 0}^{u \times q_{k}^{\prime}}
\end{array}\right\} \quad \forall j \in \mathbb{N}_{1}^{v}
\end{aligned}
$$

Similar to Section 3, the parameter set update law (25) and admissible state tube description in Proposition 18 constitute a rigorous and computationally tractable framework for model adaption and robust constraint satisfaction in receding horizon control algorithms. For systems with time-varying parameters, the MPC algorithm presented in Section 3.4 remains the same, however with the update of the membership set $\Theta_{k}$ in step (ii) replaced by (25) and the admissible set $\mathbb{D}\left(x_{k}, \Theta_{k}\right)$ in optimization (20) replaced by

$$
\mathbb{D}_{t}\left(x_{k}, \Theta_{k}\right)=\left\{\mathbf{d}_{N \mid k} \mid(15 \mathrm{a}),(28),\left(z_{N \mid k}, \alpha_{N \mid k}\right) \in \mathbb{X}_{f}\right\} .
$$

The terminal set is equivalent to the non time-varying case, i.e. based on the entire parameter set $\Theta$ and assumed to satisfy Assumption 11.

The following proposition summarizes the relevant closed-loop properties, analogously to Theorem 14 .

Proposition 19 (Closed-loop properties) Suppose Assumptions 1, 11, 17 are satisfied. If $\theta_{k_{0}}^{*} \in \Theta_{k_{0}}$ and $\mathbb{D}_{t}\left(x_{k_{0}}, \Theta_{k_{0}}\right) \neq \emptyset$, then for all $k \geq k_{0}$ we have

(i) $\mathbb{D}_{t}\left(x_{k}, \Theta_{k}\right) \neq \emptyset$

(ii) $\theta_{k}^{*} \in \Theta_{k}$,

(iii) $x_{k} \times u_{k} \in \mathbb{Z}$

for the system (24) in closed loop with the proposed MPC control law $u\left(x_{k}\right)=K x_{k}+v_{0 \mid k}^{*}$.

PROOF. As before we prove the claim by induction.

Claim (i): First note that $\Theta_{l \mid k+1} \subseteq \Theta_{l+1 \mid k}$ since $\Theta_{l \mid k+1}=\mathcal{D}_{l}\left(\mathcal{D}_{1}\left(\Theta_{k} \cap \Delta_{k+1}\right) \cap \Theta\right) \cap \Theta \subseteq \mathcal{D}_{l}\left(\mathcal{D}_{1}\left(\Theta_{k}\right)\right) \cap$ $\Theta=\mathcal{D}_{l+1}\left(\Theta_{k}\right) \cap \Theta=\Theta_{l+1 \mid k}$. Let $\left\{u_{l \mid k}^{*}, \mathbb{X}_{l+1 \mid k}^{*}\right\}_{l \in \mathbb{N}_{0}^{N-1}}$ be an admissible input and state tube. For $l \in \mathbb{N}_{0}^{N-2}$ consider the candidate solution $\tilde{v}_{l \mid k+1}=v_{l+1 \mid k}^{*}$,
$\tilde{\mathbb{X}}_{l+1 \mid k+1}=\mathbb{X}_{l+2 \mid k}^{*}$ and $\tilde{v}_{N-1 \mid k+1}=0$. For $l \in \mathbb{N}_{0}^{N-2}$ we have

$$
\mathbb{X}_{l+2 \mid k}^{*} \supseteq A_{c l}(\theta) \mathbb{X}_{l+1 \mid k}^{*} \oplus B(\theta) v_{l+1 \mid k}^{*} \oplus \mathbb{W} \quad \forall \theta \in \Theta_{l+1 \mid k}
$$

$\Leftrightarrow \tilde{\mathbb{X}}_{l+1 \mid k+1} \supseteq A_{c l}(\theta) \tilde{\mathbb{X}}_{l \mid k+1} \oplus B(\theta) \tilde{v}_{l \mid k+1} \oplus \mathbb{W} \forall \theta \in \Theta_{l+1 \mid k}$ $\Rightarrow \tilde{\mathbb{X}}_{l+1 \mid k+1} \supseteq A_{c l}(\theta) \tilde{\mathbb{X}}_{l \mid k+1} \oplus B(\theta) \tilde{v}_{l \mid k+1} \oplus \mathbb{W} \quad \forall \theta \in \Theta_{l \mid k+1}$

where the last implication follows from $\Theta_{l \mid k+1} \subseteq \Theta_{l+1 \mid k}$. Furthermore, by Assumption 11 there exists $\mathbb{X}_{N \mid k+1}$ satisfying the last set inclusion with $l=N-1$. Since $\left(x, K x+v_{l \mid k}^{*}\right) \in \mathbb{Z}$ for all $x \in \mathbb{X}_{l \mid k}^{*}$, this proves the claim for $k+1$.

Claim (ii): Let $\theta_{k}^{*} \in \Theta_{k}$ and define $\delta \theta_{k}=\theta_{k+1}^{*}-\theta_{k}^{*}$. Let $\Theta_{k} \cap \Delta_{k+1}=\left\{\theta \mathbb{R}^{p} \mid \tilde{H}_{\theta_{k}} \theta \leq \tilde{h}_{\theta_{k}}\right\}$. Then $\tilde{H}_{\theta_{k}} \theta_{k+1}^{*}=$ $\tilde{H}_{\theta_{k}} \theta_{k}^{*}+\tilde{H}_{\theta_{k}} \delta \theta_{k} \leq \tilde{h}_{\theta_{k}}+d_{\theta} \mathbf{1}$, hence $\theta_{k+1}^{*} \in \mathcal{D}_{1}\left(\Theta_{k} \cap\right.$ $\left.\Delta_{k+1}\right)$. Since by assumption $\theta_{k+1}^{*} \in \Theta$ the result follows.

Claim (iii) is again a direct corollary of $\mathbb{D}_{t}\left(x_{k}, \Theta_{k}\right) \neq \emptyset$ and Proposition 18.

The parameter variation leads to an additional tracking error in the LMS filter, invalidating the previously proved $\ell_{2}$ gain. Hence, the certainty equivalence approach as presented in this paper should only be employed for 'slow' [26] parameter variations $d_{\theta}$ and combined with a persistently exciting input. Alternatively, this difficulty could be cirumvented by using the minmax cost (19) as presented in [23].

\subsection{Persistent excitation}

The property of persistent excitation (PE) is central to classical adaptive control design [26]. It is well known that a lack of excitation can lead for example to a drift in the parameter estimates, resulting in bursts or oscillatory behavior of the closed-loop system. Similar to the classical self-tuning regulator [31], for the presented adaptive MPC algorithm a PE condition is not necessary to prove stability. Yet it ensures convergence of the parameter estimates, thereby improving closed-loop performance compared to a robust MPC approach. In the following, we give a definition of persistent excitation, provide two relevant results on parameter convergence, and show how the assumption on PE can be verified in the setup considered in this work.

Throughout this section, we make the following linear independence assumption on the parameters, which is necessary for uniquely estimating the parameters. If Assumption 20 is violated, one or more parameters can be equivalently expressed by a linear combination of other parameters. Note that it can be rewritten as a matrix rank condition and checked easily. 
Assumption 20 The equation $\sum_{i=1}^{p} \lambda_{i}\left[A_{i} B_{i}\right]=0$ has only the trivial solution $\lambda_{i}=0$.

Definition 21 (Persistent excitation) A regressor $\left\{\Phi_{k}\right\}$ with $\Phi_{k} \in \mathbb{R}^{p \times q}$ is persistently exciting if there exist positive constants $\alpha, \beta, P$ such that for all $k_{0}$

$$
\alpha I \preceq \sum_{k=k_{0}}^{k_{0}+P-1} \Phi_{k} \Phi_{k}^{\top} \preceq \beta I .
$$

This definition generalizes the more standard definition of a regressor $\phi_{k} \in \mathbb{R}^{p}$; note that for $\Phi=\left[\begin{array}{lll}\phi_{1} & \ldots & \phi_{q}\end{array}\right]$ the term $\Phi_{k} \Phi_{k}^{\top}$ is equal to $\sum_{i=1}^{q} \phi_{i} \phi_{i}^{\top}$. If a regressor is $\mathrm{PE}$, it has been proved that, under suitable assumptions on the disturbance, gradient algorithms and the set-membership estimator converge to the true parameter.

Proposition 22 (Convergence of $\hat{\theta}_{k}$ ) Assume $w_{k} \equiv$ 0 . The true parameter $\theta^{*}$ is a globally exponentially stable fixed point of the difference equation (12) if the regressor $\left\{D_{k}\right\}$ is persistently exciting.

Proposition 23 (Convergence of $\Theta_{k}$ ) Assume $\left\{w_{k}\right\}$ are realizations of a sequence of independent random variables $\left\{W_{k}\right\}$ with support $\mathbb{W}$. With probability one as $k \rightarrow \infty$

$$
\sup _{\theta_{1}, \theta_{2} \in \Theta_{k}}\left\|\theta_{1}-\theta_{2}\right\| \rightarrow 0
$$

if the regressor $\left\{D_{k}\right\}$ is persistently exciting.

The proof of Proposition 22 follows classical results, e.g. [32, Theorem 6.4], with, as previously, a minor adaption due to the matrix notation and projection. The proof of Proposition 23 follows [3, Theorem 2.1] with again a minor difference due to the matrix notation and vector valued disturbance. The assumption on the disturbance could be weakened to the boundary of $\mathbb{W}$ being a subset of the support of $W_{k}$.

The following result enables the application of the previous two propositions within the proposed MPC framework, by providing sufficient conditions on the input such that the regressor $D_{k}^{\top}$ is persistently exciting.

Lemma 24 (Persistent excitation) Suppose Assumption 20 is satisfied and $w_{k} \equiv 0$. The regressor $\left\{D_{\underline{k}}^{\top}\right\}$ is persistently exciting if the stacked input vector $\left\{\mathbf{u}_{k}^{\top}=\left[u_{k}^{\top} \ldots u_{k-n}^{\top}\right]\right\}$ is persistently exciting.

The proof of Lemma 24 can be found in the appendix. With Lemma 24 different results to achieve a PE input in MPC can be directly incorporated into the presented setup, e.g. augmenting the cost function [18], introducing an additional constraint [24] or a two-step procedure [34].
We conclude this section by remarking that instead of $\mathrm{PE}$, a min-max cost could be used to obtain optimal Dual Control $[12,37]$. However, although this leads to optimal inputs for a given cost function, it does not necessarily guarantee parameter convergence and generally leads to highly intractable, non-convex optimization programs.

\section{Numerical example}

In this section, examples are presented to illustrate the advantages of the proposed adaptive MPC scheme. We first demonstrate the online parameter identification and constraint satisfaction in a setup where stabilization of the origin is considered. Thereafter, we extend the problem setup and discuss convergence of the parameter estimates with a persistently exciting input as well as timevarying parameters.

Example 1 Consider the second-order discrete-time linear system of the form (1) with randomly generated uncertainty matrices $A_{1}, A_{2}, B_{3}$

$$
\begin{aligned}
& A_{0}=\left[\begin{array}{cc}
0.5 & 0.2 \\
-0.1 & 0.6
\end{array}\right], B_{0}=\left[\begin{array}{c}
0 \\
0.5
\end{array}\right], \\
& A_{1}=\left[\begin{array}{cc}
0.042 & 0 \\
0.072 & 0.03
\end{array}\right], A_{2}=\left[\begin{array}{ll}
0.015 & 0.019 \\
0.009 & 0.035
\end{array}\right], A_{3}=0_{2 \times 2}, \\
& \left\{B_{i}\right\}_{i=1,2}=0_{2 \times 1}, B_{3}=\left[\begin{array}{l}
0.040 \\
0.054
\end{array}\right]
\end{aligned}
$$

$\Theta=\left\{\theta \in \mathbb{R}^{3} \mid\|\theta\|_{\infty} \leq 1\right\}, \hat{\theta}_{0}=0, \theta^{*}=\left[\begin{array}{lll}0.8 & 0.2 & -0.5\end{array}\right]^{\top}$, and an iid disturbance sequence with $w_{k}$ uniformly distributed on $\mathbb{W}=\left\{w \in \mathbb{R}^{2} \mid\|w\|_{\infty} \leq 0.1\right\}$.

The MPC parameters were set to horizon length $N=10$, cost weights

$$
Q=\left[\begin{array}{ll}
1 & 0 \\
0 & 1
\end{array}\right], \quad R=1, \quad P=\left[\begin{array}{ll}
1.467 & 0.207 \\
0.207 & 1.731
\end{array}\right]
$$

and prestabilizing feedback gain $K=[0.017-0.41]$. Separate state and input constraints $\left[x_{k}\right]_{2} \geq-0.3,\left|u_{k}\right| \leq 1$, which should be satisfied robustly, were applied to the system. An additional box constraint $\left\|x_{k}\right\|_{\infty} \leq 3$ has been introduced to determine the parameter update gain $\mu$.

Figure 1 shows the state constraint $\left[x_{k}\right]_{2} \geq-0.3$, a closed-loop state trajectory for a single disturbance sequence starting from initial condition $x_{0}=\left[\begin{array}{ll}2 & 3\end{array}\right]^{\top}$, and the predicted state tube at time $k=0$. As proven in Proposition 14, the state constraint is satisfied robustly 
for all possible predicted states and the state converges to a neighborhood of the origin. Similarly, the input constraints (not plotted) are satisfied for all $k \in \mathbb{N}$.

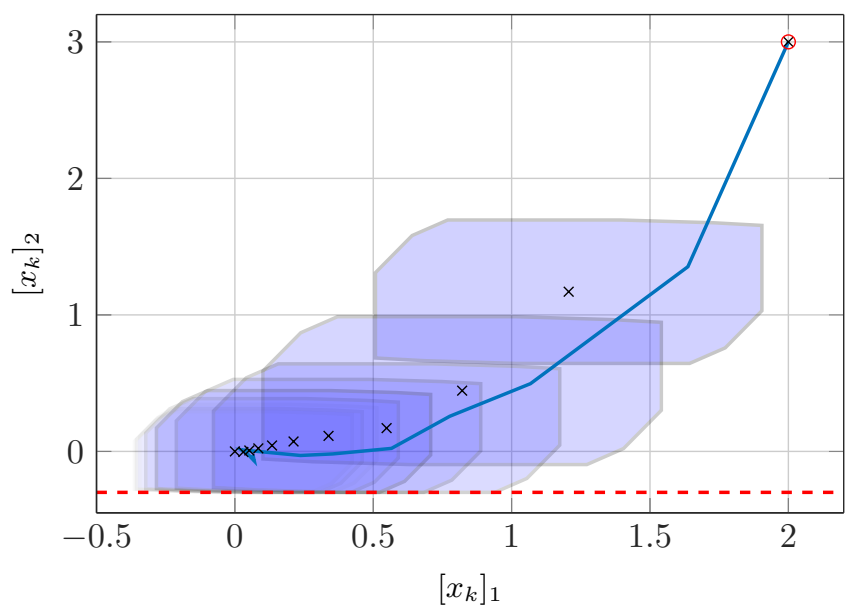

Figure 1. Realized closed-loop trajectory from initial condition $x_{0}=\left[\begin{array}{ll}2 & 3\end{array}\right]^{\top}$, predicted state tube at time $k=0$, and constraint $\left[x_{k}\right]_{2} \geq-0.3$.

To highlight the parameter estimation, the PE condition as described in Section 4.2 has been implemented, following [24], via an additional constraint on the input

$$
\sum_{l=0}^{P-1} \mathbf{u}_{k-l} \mathbf{u}_{k-l}^{\top} \succeq \alpha I
$$

with $P=n+1$ and $\alpha=2$. Starting from an initial condition $x_{0}=\left[\begin{array}{ll}0 & 0\end{array}\right]^{\top}$, the closed loop exhibits a persistently exciting regressor, with the typical cyclic state and input (Figure 2). Due to the state constraint, the center of the trajectory path is shifted to the positive orthant, such that the closed-loop state trajectory does not violate the constraint $\left[x_{k}\right]_{2} \geq-0.3$. As predicted by Proposition 23, the parameter membership set converges to a singleton (Figure 3). Given the realized state and input trajectory, falsified parameters are removed and the uncertainty set is non-increasing.

Finally, to demonstrate the capability of handling timevarying systems, in the following, the problem setup has been changed to a time-varying parameter $\theta_{k}^{*}$ with $\theta_{0}^{*}=$ $\theta^{*}$ and a bound on the variation of $\left\|\theta_{k+1}^{*}-\theta_{k}^{*}\right\| \leq 0.01$. In the simulation, the parameter has been taken to be a periodic deterministic function in time. Each parameter is increased/decreased linearly by $\frac{0.01}{\sqrt{3}}$, i.e.

$$
\left[\theta_{k+1}^{*}\right]_{i}=\left[\theta_{k}^{*}\right]_{i} \pm \frac{0.01}{\sqrt{3}}
$$

where the sign is changed upon hitting the boundary of $\Theta$. As above, the simulation has been initialized with
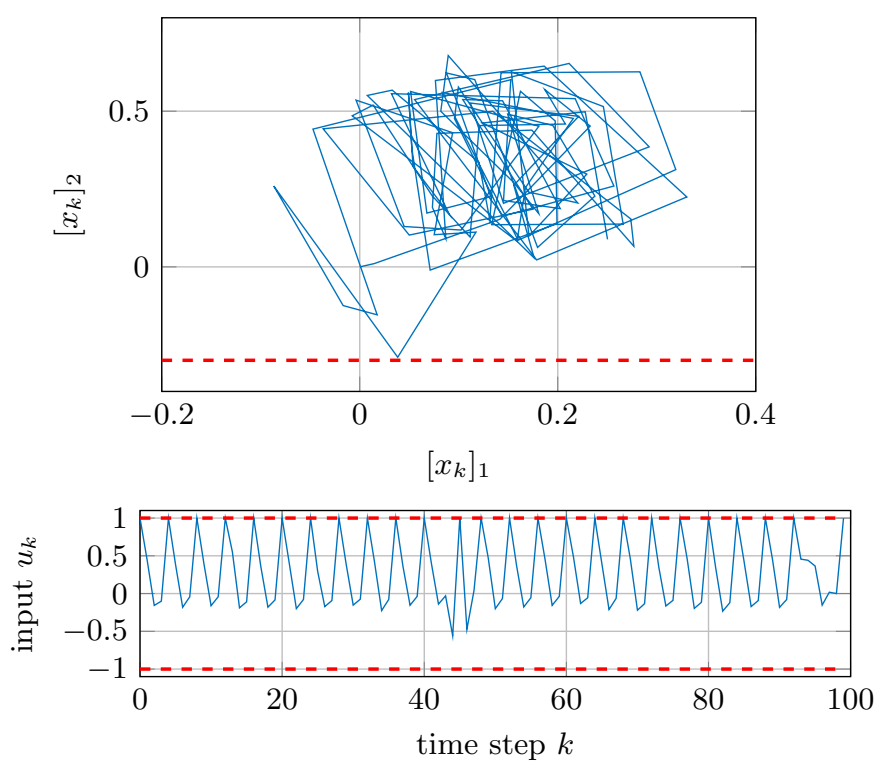

Figure 2. Closed-loop state and input trajectory with enforced PE input (solid line), state and input constraints (dashed line).
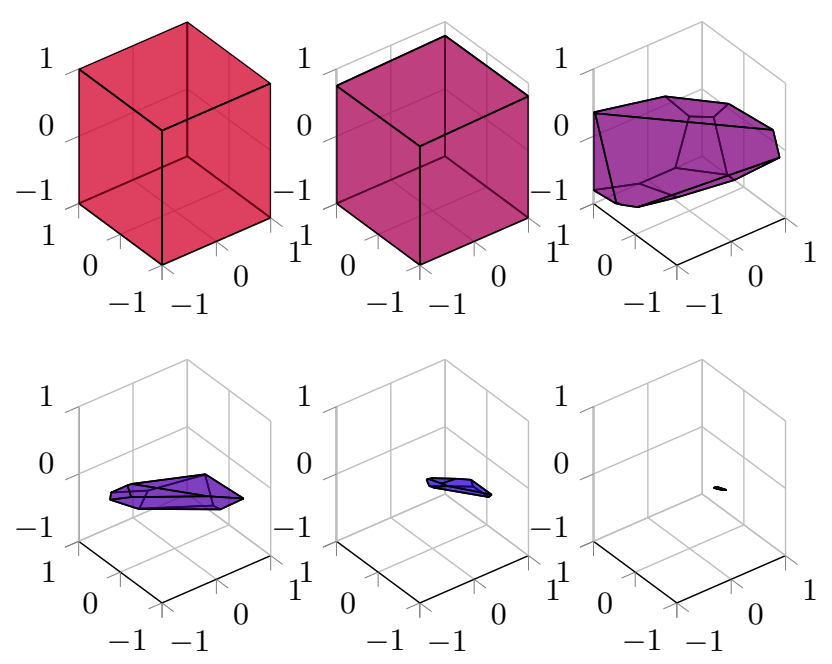

Figure 3. Parameter membership set at time steps $k \in\{0,5,25,70,120,500\}$.

$x_{0}=\left[\begin{array}{ll}0 & 0\end{array}\right]^{\top}$ and the additional PE constraint (29). Figure 4 shows the estimated parameter set at sampling times $k=1,100,200,300,400$ and 500. Instead of convergence to a singleton as in Figure 3, the parameter set varies in position, shape, and size.

The simulations were performed in Matlab with Yalmip for setting up the optimization program, which was solved using MOSEK. The median solver time (with PE constraint) reported by Yalmip was $0.068 \mathrm{~s}(0.10 \mathrm{~s})$ with a maximum of $0.095 \mathrm{~s}(0.19 \mathrm{~s})$ and minimum of $0.05 \mathrm{~s}$ on an Intel Core i7 with $3.4 \mathrm{GHz}$. Choosing $\mathbb{X}_{0}$, i.e. the shape of the tube cross sections, to be the minimal robustly forward invariant set under the local control law de- 

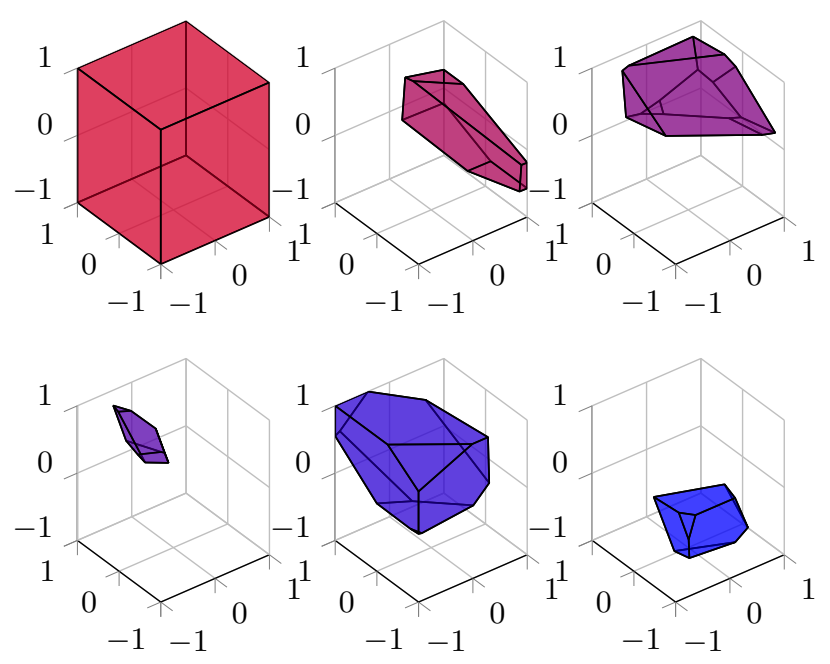

Figure 4. Parameter membership set for the system with time-varying parameters at time steps $k \in\{0,100,200,300,400,500\}$.

creases conservatism and can increase performance. Yet, due to the number of equality constraints in the MPC optimization program, a significant increase in computation time was observed with increasing complexity of $\mathbb{X}_{0}$. Furthermore, note that the scalar input allows the decomposition of the $\mathrm{PE}$ input constraint into two linear constraints, leading to two convex QP problems to be solved and compared in each MPC iteration [24].

\section{Conclusions}

A computationally tractable model predictive control algorithm with recursive parameter update has been presented that provides guarantees for closed-loop stability and robust constraint satisfaction. The requirements for stability and constraint satisfaction are considered separately. This leads to a set-membership parameter estimation scheme being employed to derive bounds on the state and input predictions whereas a Least Mean Squares filter is used to achieve a finite gain from the disturbance to the state. The online optimization to be solved is a linearly constrained quadratic program and proven to be recursively feasible. Two numerical examples are provided to demonstrate the effectiveness of the proposed algorithm.

Extensions for time-varying parameters and for PE regressors are discussed explicitly. As the MPC scheme is formulated in a modern state-space framework, the proposed setup provides a solid framework for adaptive MPC algorithms and can be easily combined with further results tailored to specific control objectives, e.g., tracking or output feedback MPC.

Compared to the classical adaptive control literature, the assumptions made are necessarily more restrictive in order to allow a robust MPC formulation. The use of less restrictive assumptions in combination with soft constraints or chance constraints are currently under investigation. In particular for the time-varying case, it would furthermore be of interest to derive bounds on the estimation error, which could then be used to relax Assumptions 8 and 11 to a parameter dependent prestabilizing feedback and terminal constraint. Finally, questions on optimal excitation of the system as discussed in [12] remain open for future research.

\section{A Appendix}

\section{A.1 Computation of the terminal region}

As shown in [8] and [29], a terminal set $\mathbb{X}_{f}$ satisfying Assumption 11 can be computed recursively by the following algorithm. With $\mathbb{X}_{0}$ and $\bar{f}$ as given above, i.e., $\mathbb{X}_{0}=$ $\left\{x \in \mathbb{R}^{n} \mid H_{x} x \leq \mathbf{1}\right\}$ and $[\bar{f}]_{i}=\max _{x \in \mathbb{X}_{0}}[F+G K]_{i} x$, let

$$
\mathbb{X}_{f}^{0}=\left\{(z, \alpha) \in \mathbb{R}^{n} \times \mathbb{R}_{\geq 0} \mid(F+G K) z+\alpha \bar{f} \leq \mathbf{1}\right\}
$$

and define

$$
\mathbb{X}_{f}^{i+1}=\left\{\begin{array}{l|l}
(z, \alpha) & \begin{array}{l}
\exists\left(z^{+}, \alpha^{+}\right) \in \mathbb{X}_{f}^{i} \text { s.t. } \\
A_{c l}(\theta)\left(\{z\} \oplus \alpha \mathbb{X}_{0}\right) \oplus \mathbb{W} \\
\subseteq\left\{z^{+}\right\} \oplus \alpha^{+} \mathbb{X}_{0} \quad \forall \theta \in \Theta
\end{array}
\end{array}\right\} \cap \mathbb{X}_{f}^{0}
$$

The sets $\mathbb{X}_{f}^{i}, i \in \mathbb{N}$ are non-increasing with $i$ and the terminal set is given by the limit for $i \rightarrow \infty$. Under the given assumptions, the sequence converges in finite time such that $\mathbb{X}_{f}=\mathbb{X}_{f}^{i}$ for some $i \in \mathbb{N}$ satisfying $\mathbb{X}_{f}^{i}=\mathbb{X}_{f}^{i+1}$.

With $\left\{\theta^{k}\right\}_{k \in \mathbb{N}_{1}^{v_{p}}}$ being the vertices of the set $\Theta,\left[\bar{h}_{x}^{k}\right]_{i}=$ $\max _{x \in \mathbb{X}_{0}}\left[H_{x}\right]_{i} A_{c l}\left(\theta^{k}\right) x$, and

$$
\tilde{\mathbb{X}}_{f}^{i+1}=\left\{\begin{array}{l}
\left(z, \alpha, z^{+}, \alpha^{+}\right) \mid \\
(z, \alpha) \in \mathcal{X}_{f}^{0},\left(z^{+}, \alpha^{+}\right) \in \mathcal{X}_{f}^{i} \\
H_{x}\left[A_{c l}\left(\theta^{k}\right) z-z^{+}\right]+\bar{h}_{x}^{k} \alpha-\mathbf{1} \alpha^{+} \leq-\bar{w} \\
\forall k \in \mathbb{N}_{1}^{v_{p}}
\end{array}\right\}
$$

the recursion (A.1) can be computed by $\mathbb{X}_{f}^{i+1}=$ $\operatorname{Proj}_{n+1}\left(\tilde{\mathbb{X}}_{f}^{i+1}\right)$ where Proj ${ }_{n+1}$ is the projection onto the first $n+1$ coordinates.

As the projection of polytopes can be computationally demanding, the recursion can be simplified through setting $z=z^{+}=0$ and determining only a suitable $\alpha$ satisfying Assumption 11.

\section{A.2 Proof of Lemma 24}

PROOF. [Lemma 24] Let $x_{k}$ be the solution of (1) with $w_{k} \equiv 0$. By [14, Corollary 2.4] $\left\{\mathbf{u}_{k}\right\}_{k}$ being PE implies 
$\left\{\left[x_{k}^{\top}, u_{k}^{\top}\right]^{\top}\right\}_{k}$ being PE. Let $d_{k}^{i}=\left[D_{k}\right]_{i}$,

$$
\Xi_{i}=\left[\begin{array}{ll}
{\left[A_{1}\right.} & \left.B_{1}\right]_{i} \\
\cdots & \\
{\left[A_{p}\right.} & \left.B_{p}\right]_{i}
\end{array}\right] \text {, and } S_{k_{0}}=\sum_{k=k_{0}}^{k_{0}+P-1}\left[\begin{array}{l}
x_{k} \\
u_{k}
\end{array}\right]\left[\begin{array}{ll}
x_{k}^{\top} & u_{k}^{\top}
\end{array}\right]
$$

Then

$$
\begin{aligned}
& \sum_{k=k_{0}}^{k_{0}+P-1} D_{k}^{\top} D_{k}=\sum_{k=k_{0}}^{k_{0}+P-1} \sum_{i=1}^{n} d_{k}^{i^{\top}} d_{k}^{i} \\
= & \sum_{k=k_{0}}^{k_{0}+P-1} \sum_{i=1}^{n} \Xi_{i}\left[\begin{array}{l}
x_{k} \\
u_{k}
\end{array}\right]\left[\begin{array}{ll}
x_{k}^{\top} & u_{k}^{\top}
\end{array}\right] \Xi_{i} \\
= & \sum_{i=1}^{n} \Xi_{i} S_{k_{0}} \Xi_{i} .
\end{aligned}
$$

Let $\underline{\sigma}$ and $\bar{\sigma}$ be the smallest and largest singular value of

$$
\left[\begin{array}{ll}
{\left[\begin{array}{ll}
A_{1} & B_{1}
\end{array}\right]_{1} \ldots\left[\begin{array}{ll}
A_{1} & B_{1}
\end{array}\right]_{n}} \\
\ldots & \\
{\left[\begin{array}{ll}
A_{p} & B_{p}
\end{array}\right]_{1} \ldots\left[\begin{array}{ll}
A_{1} & B_{1}
\end{array}\right]_{n}}
\end{array}\right]
$$

which are nonzero by Assumption 20, and define $\alpha^{\prime}=$ $\underline{\sigma}^{2} \alpha$ and $\beta^{\prime}=\bar{\sigma}^{2} \beta$. Given $\alpha I \preceq S_{k_{0}} \preceq \beta I$, we have $\bar{\alpha}^{\prime} I \preceq \sum_{i=1}^{n} \Xi_{i} S_{k_{0}} \Xi_{i} \preceq \beta^{\prime} I$, which proves the claim.

\section{References}

[1] V. Adetola and M. Guay. Robust adaptive MPC for constrained uncertain nonlinear systems. International Journal of Adaptive Control and Signal Processing, 25(2):155-167, 2011.

[2] A. Aswani, H. Gonzalez, S. S. Sastry, and C. Tomlin. Provably safe and robust learning-based model predictive control. Automatica, 49(5):1216-1226, 2013.

[3] E.-W. Bai, H. Cho, and R. Tempo. Convergence properties of the membership set. Automatica, 34(10):1245-1249, 1998.

[4] D. S. Bayard and A. Schumitzky. Implicit dual control based on particle filtering and forward dynamic programming. International Journal of Adaptive Control and Signal Processing, 24(3):155-177, 2010.

[5] A. Bemporad, M. Morari, V. Dua, and E. N. Pistikopoulos. The explicit linear quadratic regulator for constrained systems. Automatica, 38(1):3-20, 2002.

[6] A. Ben-Tal, L. E. Ghaoui, and A. Nemirovski. Robust optimization. Princeton Series in Applied Mathematics. Princeton University Press, 2009.

[7] R. R. Bitmead, M. Gevers, and V. Wertz. Adaptive Optimal Control, The Thinking Man's GPC. Prentice Hall, 1990.

[8] F. Blanchini and S. Miani. Set-Theoretic Methods in Control. Systems \& Control: Foundations \& Applications. Birkhäuser Boston, 2nd edition, 2015.
[9] M. Bujarbaruah, X. Zhang, and F. Borrelli. Adaptive MPC with chance constraints for FIR systems. ArXiv e-prints, abs/1804.09790, 2018.

[10] L. Chisci, A. Garulli, A. Vicino, and G. Zappa. Block recursive parallelotopic bounding in set membership identification. Automatica, 34(1):15-22, 1998.

[11] S. Di Cairano. Indirect adaptive model predictive control for linear systems with polytopic uncertainty. In Proceedings of the American Control Conference, pages 3570-3575, Boston, MA, July 2016.

[12] A. A. Feldbaum. Dual-control theory I. Automation and Remote Control, 21:874-880, 1961. Translated from Avtomatlka i Telemekhanika, 21(9), 1960.

[13] E. Fogel and Y. Huang. On the value of information in system identification - Bounded noise case. Automatica, 18(2):229$238,1982$.

[14] M. Green and J. B. Moore. Persistence of excitation in linear systems. Systems 83 Control Letters, 7(5):351-360, 1986.

[15] M. Guay, V. Adetola, and D. DeHaan. Robust and Adaptive Model Predictive Control of Nonlinear Systems. Control, Robotics and Sensors. Institution of Engineering and Technology, 2015.

[16] B. Hassibi, A. H. Sayed, and T. Kailath. LMS is $H^{\infty}$ optimal. In Proceedings of the Conference on Decision and Control, pages 74-79 vol.1, Dec 1993.

[17] T. A. N. Heirung, B. Foss, and B. E. Ydstie. MPC-based dual control with online experiment design. Journal of Process Control, 32:64-76, 2015.

[18] T. A. N. Heirung, B. E. Ydstie, and B. Foss. Dual adaptive model predictive control. Automatica, 80:340-348, 2017.

[19] T. H. Kim and T. Sugie. Adaptive receding horizon predictive control for constrained discrete-time linear systems with parameter uncertainties. International Journal of Control, 81(1):62-73, 2008.

[20] E. D. Klenske, M. N. Zeilinger, B. Schölkopf, and P. Hennig. Gaussian process-based predictive control for periodic error correction. IEEE Trans. Control Syst. Technol., 24(1):110121, Jan 2016.

[21] B. Kouvaritakis and M. Cannon. Model Predictive Control: Classical, Robust and Stochastic. Springer International Publishing, Cham, Switzerland, 2016.

[22] W. Langson, I. Chryssochoos, S. V. Raković, and D. Mayne. Robust model predictive control using tubes. Automatica, 40(1):125-133, 2004.

[23] M. Lorenzen, F. Allgöwer, and M. Cannon. Adaptive model predictive control with robust constraint satisfaction. In Proceedings of the IFAC World Congress, pages 3368-3373, Toulouse, France, 2017

[24] G. Marafioti, R. R. Bitmead, and M. Hovd. Persistently exciting model predictive control. International Journal of Adaptive Control and Signal Processing, 28(6):536-552, 2014.

[25] D. Q. Mayne. Model predictive control: Recent developments and future promise. Automatica, 50(12):2967-2986, 2014.

[26] K. S. Narendra and A. M. Annaswamy. Stable adaptive systems. Dover Publications, 2005.

[27] C. J. Ostafew, A. P. Schoellig, and T. D. Barfoot. Conservative to confident: Treating uncertainty robustly within learning-based control. In Proceedings of the IEEE International Conference on Robotics and Automation, pages 421-427, May 2015.

[28] S. Qin and T. A. Badgwell. A survey of industrial model predictive control technology. Control Engineering Practice, 11(7):733-764, 2003. 
[29] S. V. Raković and Q. Cheng. Homothetic tube MPC for constrained linear difference inclusions. In Proceedings of the 25th Chinese Control and Decision Conference, pages 754761, May 2013.

[30] S. V. Raković, B. Kouvaritakis, R. Findeisen, and M. Cannon. Homothetic tube model predictive control. Automatica, 48(8):1631-1638, 2012.

[31] K. J. Åström and B. Wittenmark. On self tuning regulators. Automatica, 9(2):185 - 199, 1973.

[32] K. J. Åström and B. Wittenmark. Adaptive Control. Dover Publications, 2nd edition, 2008.

[33] J. B. Rawlings, D. Q. Mayne, and M. M. Diehl. Model Predictive Control Theory and Design. Nob Hill Publishing, 2nd edition, 2017.

[34] M. Tanaskovic, L. Fagiano, R. Smith, and M. Morari. Adaptive receding horizon control for constrained MIMO systems. Automatica, 50(12):3019-3029, 2014.

[35] M. Tanaskovic, D. Sturzenegger, R. Smith, and M. Morari. Robust adaptive model predictive building climate control. In Proceedings of the IFAC World Congress, pages 18711876, Toulouse, France, July 2017.

[36] S. Veres and J. Norton. Predictive self-tuning control by parameter bounding and worst-case design. Automatica, 29(4):911-928, 1993.

[37] S. M. Veres. Adaptive control by worst-case duality. In C. Bányász, editor, 5th IFAC Symposium on Adaptive Systems in Control and Signal Processing, IFAC Postprint Volume, pages 73-78. Pergamon, Oxford, 1995.

[38] S. M. Veres, H. Messaoud, and J. P. Norton. Limitedcomplexity model-unfalsifying adaptive tracking-control. International Journal of Control, 72(15):1417-1426, 1999. 